\title{
Tutorial chat: a case study of synchronous communication in a learning environment
}

\author{
Malcolm Rutter* \\ Napier University, UK
}

\begin{abstract}
This case study describes the use of a web-based synchronous chat application, run during computing tutorials. The chat room was moderated by a paid demonstrator, who assisted and encouraged students whenever possible. Most of the discussion was banter, which acted as a lubricant for relevant factual communication. Students were permitted a degree of anonymity, but nevertheless learned to treat the facility in a useful manner. The application and its mode of employment were found to be both motivating and supportive. Use was evaluated by questionnaire and an analysis of student input. As a result of the experience, it is suggested that abuse will certainly occur and may be minimised by technical improvements, but never eliminated. The Rogerian approach adopted was found to have transferred emphasis from teaching a topic to the learning of generic skills.
\end{abstract}

\section{Introduction}

This article describes the use of a chat application in computing tutorials. The application itself was sufficiently interesting to the students to be motivating. Once attracted into the online community, students began to share experience in a way that forwarded the aims of the module. This would be justification enough, but the difficult environment in which the tutorials operated made the exercise even more valuable.

This paper's purpose is to report experience gained from the exercise, for the guidance of those considering similar activities. Lessons are drawn that could guide the modification of the application and its use more successfully in future

\section{Background}

Although the use of asynchronous online communication environments has been quite extensively researched, less has been written about synchronous environments.

\footnotetext{
* School of Computing, Napier University, 10 Colinton Road, Edinburgh EH10, UK.

Email: malcolmrutter@ieee.org
} 
These are distinctive in several ways. Wright et al. (2004), for example, writing regarding a web-based forum, report that students do not appear to use threads in a useful way. It is the nature of the pooling of knowledge that contributions are often not conveniently clustered into a topic thread. For this reason, non-threaded chat structures have been adopted in some courses (including that described here). This decision is not without its drawbacks; Pimintel et al. (2003) report a phenomenon that they call 'co-text loss', concerning confusion about which statement relates to which thread. It is not obvious to a contributor that their statement could have meaning in a number of possible contexts, and this causes ambiguity.

Although synchronous environments are distinctive in several respects, there are also a number of contextual issues that influence their success that they share with asynchronous environments. For example, although the idea of blended learning is now widespread, this mostly considers mixing asynchronous web-based materials with face-to-face teaching. The use of an online proximate community (Wright et al., 2004) within a tutorial is an idea not hitherto encountered.

The topic of alienation in large classes has also been well reported. Preston and Shackelford (1998) observe that students may feel anonymous in large classes, where instructors have difficulty in appreciating feedback. Among other methods, they have successfully used newsgroups in order to get discussion and feedback on the module they teach. A differing view is advanced by Kay (1998). Kay agrees that alienation in large classes can lead to lack of attention and ultimately attrition, but he advocates more personal solutions, such as laboratory partners, group work and peer testing. Interestingly, he is pessimistic about electronic communication systems on the grounds that much interpersonal communication is non-verbal, although such concerns are less visible in contemporary literature on this topic.

One feature that many students find attractive is the ability to use a nickname and icon as a mask to hide one's true identity. Millen and Patterson (2003) argue that accountability encourages politeness and trust. They describe an online proximate community with a policy that enforces 'legal name' identification of participants. Farkas et al. (2002) point out that there are circumstances when anonymity is desirable, the reviewing of papers for example. They describe an 'electronic Editorial Board' in which users are accountable to a trusted mediator. Within this context, they are free to adopt multiple personas to other participants. In conventional online communities a participant may be barred and yet log on later as another persona; however, barring in the Farkas community affects all of a participant's personas, present and future. Both Farkas et al. (2002) and Preece (2000, p. 155) also comment that other participants may infer links between multiple personas from linguistic style. Preece (2000, p. 197) herself has a more optimistic view of anonymity: she acknowledges that anonymity does permit irresponsible behaviour and it does happen, but that usually it does not. Whereas in real life, she comments, the bystander effect may inhibit observers from helping a person in difficulties, online participants are more forthcoming.

Another recurrent issue in the literature concerns the role of moderation. Preece, for example, commented (2000, p. 228) that online communities die if left to fend for themselves. The long-term support of a community is often entrusted to one or more 
moderators (p. 229), and she outlines the numerous tasks that such a person may carry out (Preece, 2000, p. 83). The importance of moderation is easy to establish. For example, Light et al. (2000) report an online forum used as a seminar group. In a paper that should be required reading for those using computer-mediated communication, Light et al. describe an environment in which the participants had no preparation or advice, nor was it moderated. It is a story of good students who unwittingly attracted painful criticism, of online vandalism by a pair of males who set out to insult participants and disrupt the forum, and of a community so socially damaged that it had difficulty functioning usefully. The conclusions of the paper are that rules, advice and moderation are necessary and that, if necessary, disruptive students may be warned or withdrawn.

Given these issues - of communication, identity and moderation - it is somewhat surprising that a Rogerian (i.e. person-centred) approach to facilitation has not been more widely studied (Mahoney \& Baker, 2002). To address this gap, this paper explores a case in which this approach was adopted. This experience will be analysed in order to assess the possibility of adopting this model to support teaching more widely.

\section{The case context: a Rogerian approach to tutorial support}

The environment in which this study was conducted was a 500-seat computing facility, designed for ease of administration. The difficulties experienced in trying to use the facility for education are already documented (for example, Buckner \& Davenport, 2002). The environment's acoustics make it difficult to communicate with a group of students as a whole. Tutorial sessions are often invaded by students, singly or in groups, seeking free machines to do other work. The approach adopted in the module under consideration ( 43 students) was to give the group printed sheets of tutorial work. Individual help was available on request.

Students were observed communicating with one another using Microsoft Messenger. It was felt that this medium could be incorporated into the educational experience. Accordingly, students were offered a synchronous communication application (chat room) to use during tutorials.

The chat facility was provided to try to establish a sense of group identity for those on the module, independent of space. These were Scottish Credit and Qualifications Framework (SCQF) level nine direct entrants (the year prior to the honours year), and it was hoped to encourage a group spirit and a collaborative approach to learning. Finally, it was hoped to motivate the students, so as to increase attendance and participation. Given the importance attributed to facilitation in the literature, a moderator was provided for the online group.

Informal discussions with colleagues had highlighted that students would not or could not stick to a predetermined topic in online chat and forums. It was decided to accept this predicted anarchy and work with the students rather than against them. This has strong parallels with the operation of therapeutic juvenile psychotherapy groups. Foulkes and Anthony (1965, p. 210) state: 
During the early sessions, when the children discover the opportunity afforded them to express themselves, the level of noise can be considerable. Interpretations are apparently 'lost' within the maelstrom. Later, at quieter moments, they come back from the group, having been heard. Experience shows that little that the therapist says goes unperceived.

The aim of the module leader was to foster a learning community of the kind described by Rogers (1967, chapter 15), in which the facilitator provides respect and encouragement and the students do the active learning. This way of interacting was found to have encouraged both participation and the sharing of experience within therapeutic contexts and has been advocated within traditional educational practice.

This case was considered to be worth studying because the problems of the environment were a feature of most tutorial work undertaken in the school, and because a solution of this kind would be a relatively cheap enhancement to the learning environment. The aims of the research, then, were to monitor and report on the success or otherwise of the chat facility, to provide guidance in improving this as an aid to learning and to offer guidelines to those attempting a similar approach.

\section{Methodology}

The application on which the study was based was chosen from a number of available free packages. It was chosen for its technical simplicity-it used the PHP language, which was known to operate on the School's web server. Often a forum or a chat room would use a database, such as MySQL; this application was even simpler, storing input in a text file on the server. A non-threaded application was chosen.

Apart from informal discussions with individual students, two methods of monitoring were chosen. The application offered the ability to print off the contents of the stored file, containing the last 150 utterances. This was analysed for content, recording the quantity and topics of the student utterances. The second feedback method was a questionnaire, administered to the students late in the module. The questionnaire allowed quantitative analysis, but also contained open-ended questions to collect student ideas.

Further research methods were considered but not used. A comparison of module marks with and without the chat facility, follow-up studies of the cohesiveness and success of the cohort or even the routine module student satisfaction survey might have demonstrated that the approach was superior or redundant. These routes were not taken because of the variability of cohorts and the statistical noise on the data.

\section{Technical provision}

The chat package selected was a linear or unthreaded chat application, Simple Chat 1.3 (Anonymous, 2004), as shown in figure 1. (The Simple Chat package is currently available free as BlaB! lite 2.2.) It ran on the web site of the School of Computing, which was used as a repository for the rest of the module material. Before entry, students were asked to choose a nickname of eight characters or less; many chose to base this on their own name. They were also offered a selection of identifying icons. 


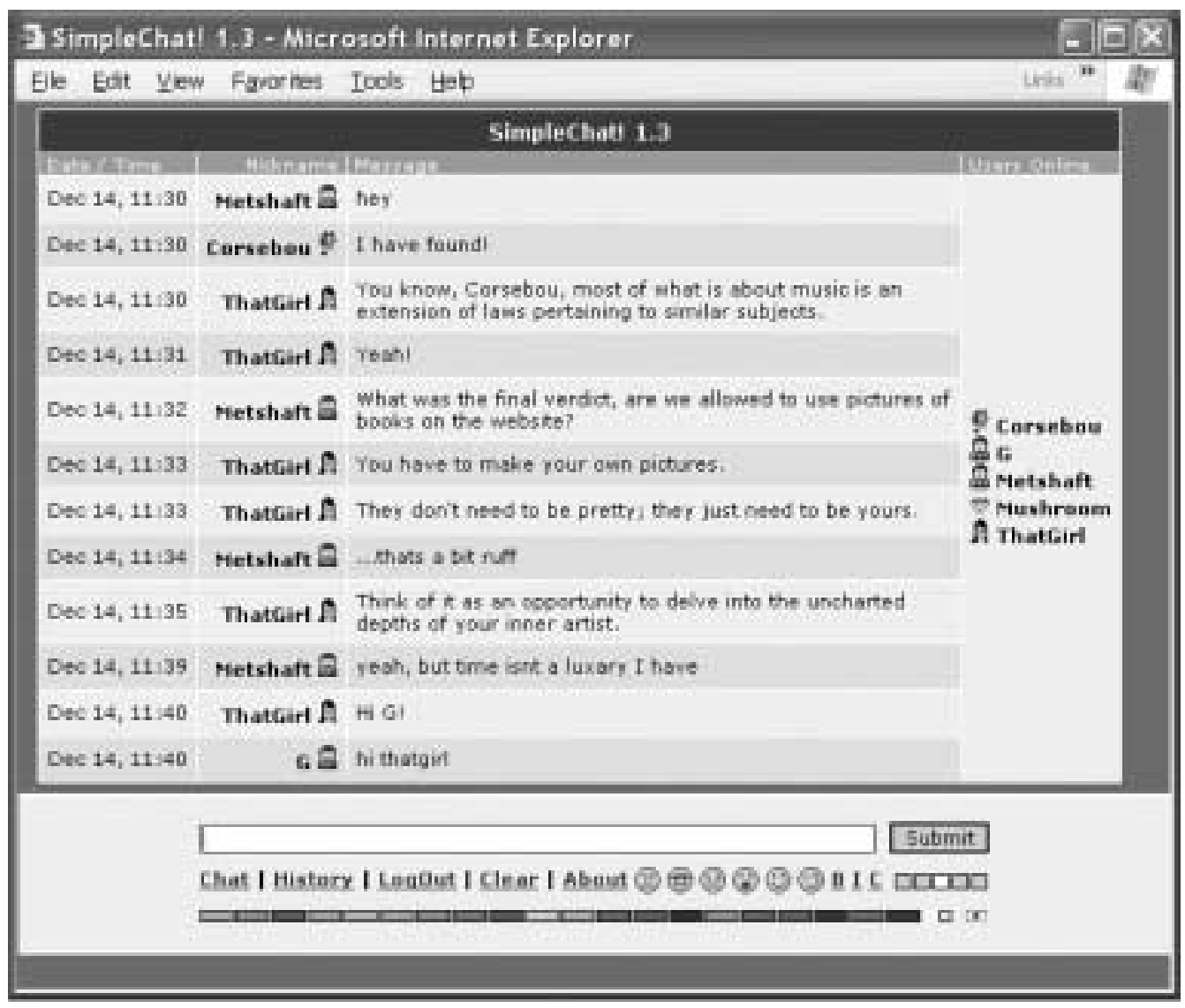

Figure 1. Screen shot of the chat application. The bottom frame is for composing messages. The top frame shows the ongoing dialogue. The column on the right shows who is online at the moment.

Note the icons, some of which imply gender

Male and female icons were available, but also ungendered cartoon characters. A feature of the application was a side panel showing the nicknames and icons of all students online at the time. If not erased, the file system would store only the last 150 inputs. Experience showed that this represented almost two hours of operation.

The chat room was manned during tutorials. Initially the module leader ran it, but eventually a student, employed as a demonstrator, asked if she could take over. She welcomed students as they came online, took part in the chat and offered advice and encouragement where appropriate. The (mostly male) students commented that they were more comfortable with a female than a male.

\section{Results}

The chat room was active throughout most of the two-hour tutorial. Between 4 and 15 of the 43 students on the module used the facility at any one time. It appeared that 
the success of the application had a circular effect, the large number of users attracting even more. One student commented in class that she had visited the chat room out of hours but was disappointed to find herself alone. (The application had also been left running experimentally over the summer and during this time only one person, from somewhere on the Internet, had left a message to say he/she had visited.)

Discussion was mostly frivolous, covering diverse topics such as books read, films seen and good places to dance on the weekend. There was also surreal input. Students coped with the linear nature of the presentation by holding multithreaded conversations anyway. At any one time there could be as many as three independent conversations interleaved with one another. It was believed that the frivolous chatter was a way of claiming the medium and establishing a level of trust. Among the banter, there was space for students to discuss the module. Some shared their successes, supplying the URLs of their web sites. Some asked about problems with the formal tutorial exercises (running in parallel as the 'main' work). There were queries about the wording of the assignment specification. Some asked for help with technical difficulties with their web sites - they said they would rather ask online than raise their hands to ask for help from a physical demonstrator. One student, on seeing some of the very successful web sites, confided his feelings of inadequacy and received reassurance from the moderator.

In the first week, while the group was getting used to the environment, some of the international students posted messages in French and in Spanish. One of those messages contained a mild swear word. Following that, the whole group settled down to communication in English. Expletives were confined to the 'sh ... t' forms acceptable in everyday journalism.

It was possible for a student to miss the tutorial through illness but nevertheless log on from home. This was observed once in week nine.

\section{Analysis of transcripts}

The software discarded all but the last 150 utterances in the chat room. The students had not discovered how to make long statements as one utterance, so one statement could go on over a number of utterances.

The total number of utterances recorded over 10 weeks was 1224 . Times were recorded automatically, allowing the calculation of an average of 94.2 utterances per hour. This varied from the record rate of 250 utterances per hour of the first week to the low 47 utterances per hour on the week the first assignment was due in.

The transcripts were read and common themes noted. Then they were analysed to measure how frequently these topics cropped up, and in what patterns (Figure 2).

The most important question for the module leader was 'how often did the students share ideas about the module' (Figure 3)? Three hundred and twenty-one utterances were counted, averaging $26 \%$ of the total. There was a peak at $47 \%$ the week the students got their web servers to work, which was a tricky operation. Another peak came in week eight when the students were struggling to programme in XHTML. The actual number of utterances was lower, possibly because attention was 


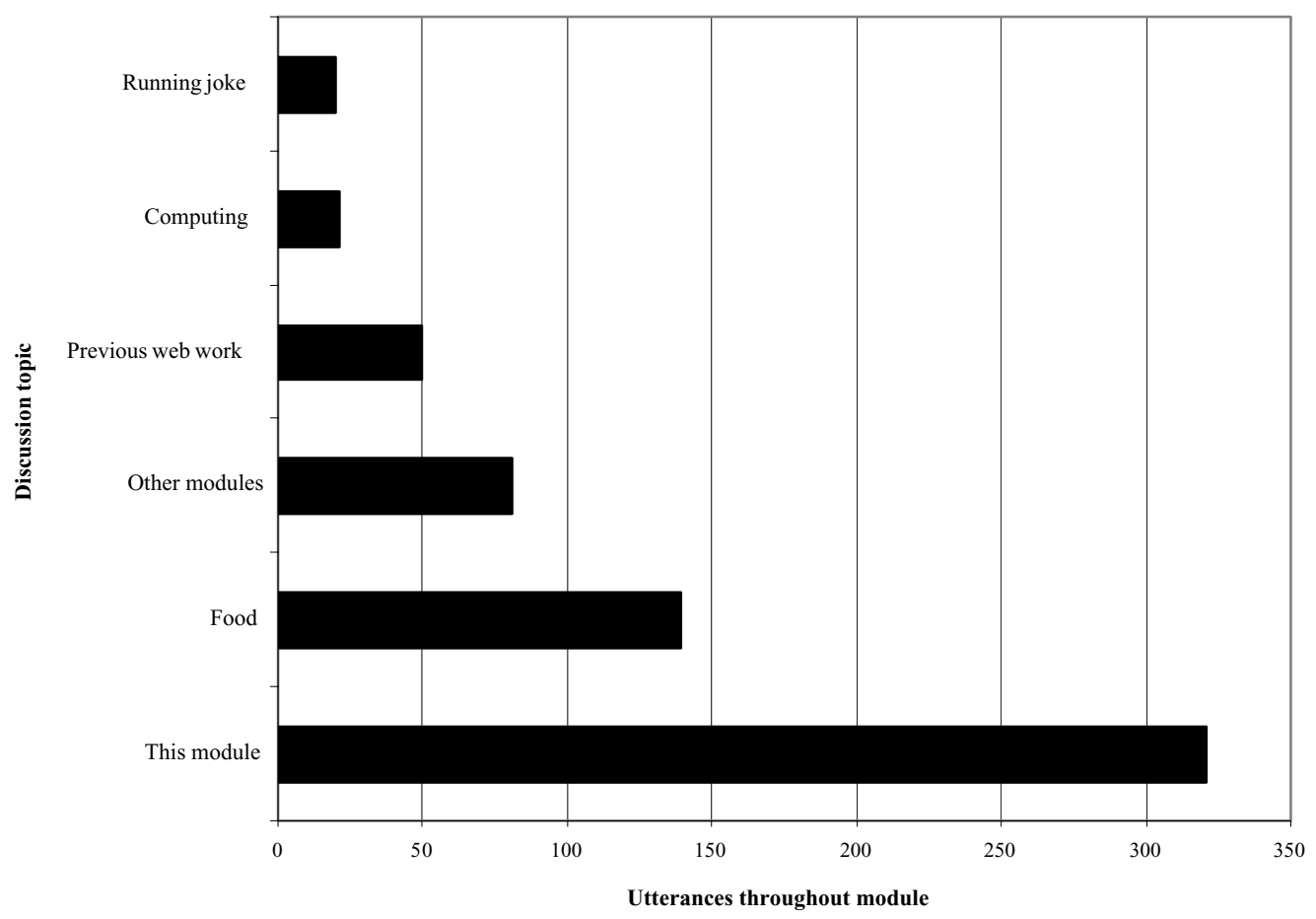

Figure 2. The frequencies of utterance of topics throughout the module

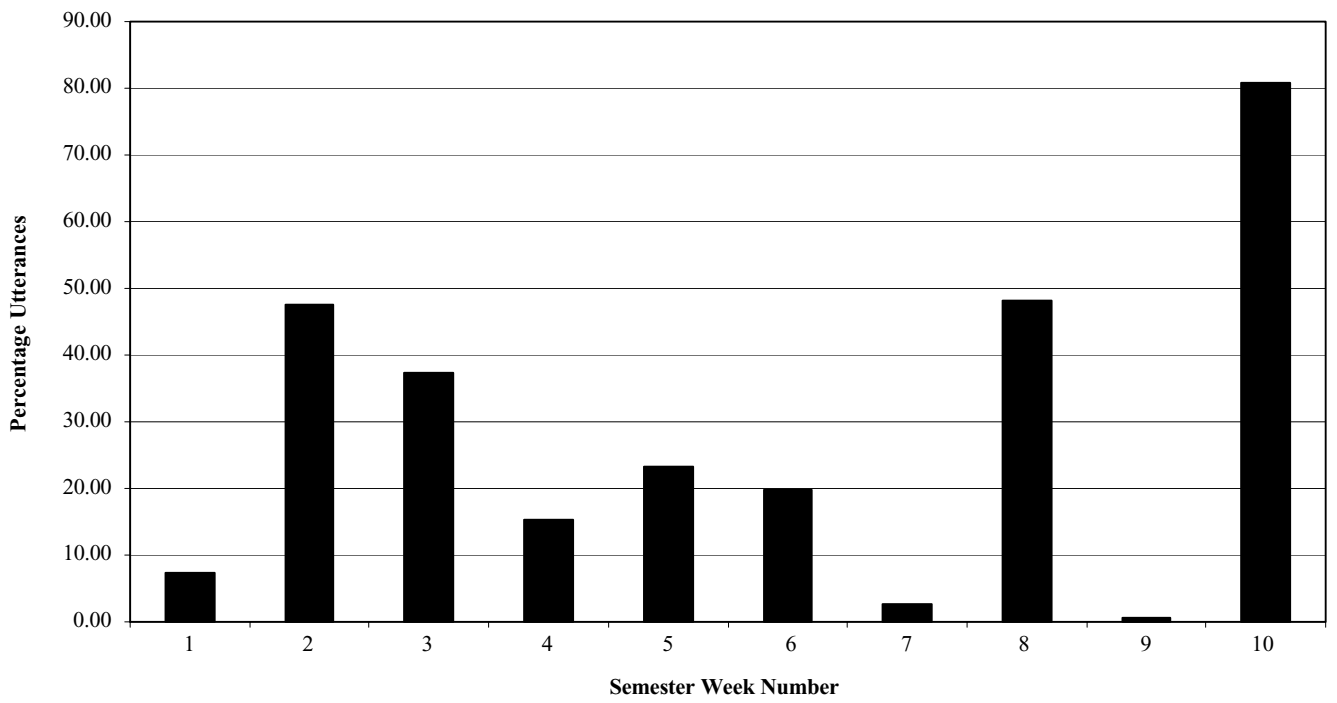

Figure 3. Percentage of utterances relevant to the module, versus week number 
absorbed by the programming task, but the percentage of relevant utterances was $41 \%$. The highest peak of $81 \%$ resulted from a tutorial examining bad practice in web design, as students reported their finds.

Apart from discussion of the work of the course and the module, less than $2 \%$ of all the utterances were about the general topic of computing. There was, for instance, a discussion about the wisdom of installing a particular operating system upgrade.

Another topic was that of university assignments not related to this module. Normally this topic remained at $7 \%$ of the utterances, although there was a surge in week nine when a student reported printing problems and others offered advice and support ( $41 \%$ of utterances that week were classified as being about 'other coursework').

Social statements were considered significant, in that they were a measure of the cohesiveness of the group. These ranged from simple greetings when a new member came online to detailed instructions about how to find a karaoke night. Social activity also overlapped other activities, such as when students asked for and offered advice. Most utterances were to the moderator or to the group in general. A smaller but significant number of statements were made between individual students.

At the start of the module, some students showed the online group previous web work that they had done and discussed it with the others. These discussions accounted for 50 utterances. One surreal running joke ran over five weeks, accounting for 20 utterances. The introduction of this topic may have been symbolic of the students owning and accepting the chat facility as theirs to use as they wished.

Finally, the topic of food was very important to the students. The tutorial had been timed to run up until lunchtime (11:00-13:00). Of all the utterances recorded throughout the module, more than $11 \%$ were about food.

\section{Analysis of questionnaires}

A questionnaire was administered to students and the results collated (Appendix 1). Seventeen students responded.

Fourteen students had logged in to the chat facility at one time or another. Those that had not, said that they had awarded it a low priority compared with the module assignment and official tutorial work. Tolmie and Boyle (2000) and Salmon (2002) maintain that students will only use online tools when there is a good reason to do so.

Six students had logged in but not contributed. Unlike those lurking in a forum or newsgroup, their presence would have shown on everyone's screen. This would have earned them a welcoming greeting from the moderator. The lurkers gave various reasons for non-contribution. Most said that they had had nothing to say. An international student reckoned that his English was too slow to cope with the pace of the discussion. One student confided that he was afraid of being laughed at by the others. Light et al. (1997) pointed out that the fear of revealing one's opinions for scrutiny would discourage active participation.

Most (four of six) of the lurkers reported benefiting from sharing the experiences of those who contributed. One said that seeing others sharing difficulties helped his 
own feelings of inadequacy. Light et al. (1997) and Wilson and Whitelock (1998) have reported the benefit of this vicarious form of learning.

Many of those who had contributed online commented on the good atmosphere and friendly conversation. One-half of the contributors had used the chat room to ask for information. Benefits reported started at a factual level ('how to upload a web site') but went on to meta-levels ('I knew I only needed to ask for help'). There was at least one example of learning a soft skill:

I have learned that taking the time to ask when you are stuck is better than not asking ... and the chat is anonymous so there is more confidence gained if you are not articulate in asking face-to-face questions.

Asked to comment on what social gains they may have made, there were three types of answer. Some said that there were no perceivable social benefits. Some said they had focused on using the facility for functional, factual purposes. A third group said they had been drawn in, in search of facts, but had thereby got to know others.

The module leader was concerned whether the anonymity implied by the use of nicknames helped or hindered. A minority (2 of 12) said they would have preferred to know to whom they were talking. Most (9 of 12) wanted to keep the nicknames. Some said nicknames were fun. Others said that they helped with shyness in the early stages; if a student asked a silly question, their anonymity would protect them. One commented that eventually everyone got to know who everyone else was anyway - the anonymity had acted as a crutch only in the early weeks.

Students were asked whether the chat room was worth keeping in future deliveries of the module. Everyone who responded to that question answered in the affirmative. Reasons given were that it integrated the class, was fun and gave quick access to information.

\section{Counter-examples}

Not everyone shared the enthusiasm for chat rooms. Those who had not used a chat room pointed out that it was not assessed and that it was not an official part of the module. One commented that a lot of the chat was trivial light conversation. Another said she had used it in the first week, because that was a tutorial exercise, but never since. A user said that he had never learned anything of relevance from the facility, while quite a few said they did not regard it as socially useful.

Some students were observed to be using the MSN Messenger application instead. They explained that they had formed a study group of their own. They regarded the chat facility as purely for the Web Design module, whereas they used Messenger for the whole programme of study. Furthermore, Messenger could be ignored until a colleague had something to say, when a message would appear in the system tray; the chat application only gave an audible warning, and sound was always turned off in the laboratories. 


\section{Problems}

The module is delivered every semester. An incident occurred in a later delivery to second-year students: in one tutorial group, in week one, two students maintained a long interaction, insulting each other in an obscene way. The demonstrator asked that they would stop, both online and by calling out to the group, but they did not. The module leader interpreted this as a combination of the excitement that grips many students when encountering chat for the first time and the 'flame effect', in which users are led to express anger very forcefully (Preece, 2000, p. 83). This presented a philosophical challenge: demonstrations of power by the lecturer would have been counter-productive, since the aim was to encourage and empower the students. The module leader took the opportunity of the next lecture to explain to all the students that the chat room was intended for them to use, but that he himself faced constraints. There was a risk that he might be asked to remove the application.

The costs of this facility are also an issue. The chat facility will not work well without a facilitator, costing $£ 8.80$ per hour. For the whole 13 -week module this works out to $£ 4.40$ per student. It should be noted that only a subset of students seemed to benefit from this, since styles differ and not every student uses the facility.

\section{Discussion}

One view of a university's role is that of inculcating generic transferable skills that will stay with the students for the rest of their lives (Brass \& Pilven, 1999). Thus a programme in computing will serve to teach generic skills against a background of computing. In this context, a Rogerian approach should develop self-awareness and a Rogerian perspective in participants. One of the most important skills learned was that of sharing problems with others and the good feelings that come from helping colleagues. It is hoped that this will encourage mutual support and the enrichment of the learning environment, leading in turn to better learning. Another advantage is that both online chat and a Rogerian approach are very attractive to students; this extra motivation would serve to increase work input.

As a result of experiences with the chat room application, it was decided to continue to use it for future delivery of the module. The question of justifying this decision is a challenging one, however. It is possible to address the problem of measuring the effectiveness of education, to see whether value for money is being obtained. Certainly, it was the experience of Halfpenny and Wellings (2001) that virtual seminars do not reduce the resources needed in education, since they augment rather than replace the existing systems. If this is so, it may be more appropriate to explore changes in the quality of the student experience and resulting mindset towards education.

The module in question is franchised internationally, but the system would need to be rethought before using it on large cohorts in parallel. It does not appear to be scaleable in its present form. However, the advantage of an Internet-based facility is that the person moderating it does not need to be in the same physical location. They 
could be at an office desk or on a different site. Time zones permitting, one institution could host tutorials for partner colleges throughout the world.

The observation of the parallel use of MSN Messenger should not have been a surprise-its use is common throughout the facility. If experiences of chat have encouraged students to form online study groups, then this has to be an enhancement of the educational experience. Previous experiences of (offline) spontaneous study groups have shown that they are well be worth fostering. The module leader must now consider whether Messenger is a more appropriate application to adopt.

Sometimes the most important outcome of a piece of research is the refining of the questions to be addressed (Baskerville, 1999). One such question concerns the use of nicknames. These were intended to release inhibitions and encourage a greater freedom. The anonymity they provide might prevent the growth of real relationships through this medium. One student even went so far as to collect a table matching names to nicknames, either through curiosity or a deeper need.

The importance of a moderator cannot be overemphasised. It is the job of the moderator to take the reticence or enthusiasm of the students' first encounter with the chat room and maintain the warmth and interest. Rogerians speak of unconditional positive regard and illuminating the humanity of the input of participants. A more pragmatic magnet is the use of the moderator as a source of advice on the ongoing work of the module.

There was a powerful response in week nine, when a student reported difficulties in printing a document. This is evidence that a group identity had formed, as one would expect with groups that meet and share. What is not so certain is the chat room's contribution to this identity.

The policing of a chat room is a delicate subject. Preece (2000, p. 95) comments that communities can die under a deluge of flaming and spam, but that a long list of rules is very discouraging. At the moment, the chat room is completely unprotected; in theory, anyone with Internet access can maliciously disrupt communications. The fact that this has only happened once does not guarantee that it will not become a problem; indeed, it suggests that it will recur. Technology can help, although it may not be a complete answer. Firstly, there are parts of the university network into which students need to $\log$ in. Students could keep their online anonymity, as long as they were also accountable. This might deter mischief. A common solution in many chat rooms is for the moderator, in the last resort, to be able to exclude ('boot') a user with a certain IP address. Swear-word detection and removal is another common feature in chat room software (on one site, detected swear words are automatically replaced with the names of fruit and vegetables).

\section{Conclusions}

The research aim was to monitor the project, to determine how it could be improved and to provide guidance for others to follow a similar approach. These research aims were met in so far as qualitative evidence was gathered that indicated some students were benefiting educationally and there was some evidence for the social skills of the 
group having developed. It was not found possible to quantitatively determine the profitability of the medium. Support, both online and in class, was found to be essential to the success of the exercise.

For others following this route, the trade-off between anonymity and accountability needs to be thought out carefully beforehand. The lack of security in the simple application allowed misuse to occur; indeed, in any application, misuse will certainly occur as the students test and experience the new medium. Technology can help a little, in the provision of restricted access, accountability, temporary barring and the automatic removal of untoward words, but it is only part of the answer. There is evidence for the importance of the support given both online and in class. It was hoped that fostering a spirit of collaboration in the students might encourage a more successful approach to learning. In fact, the Rogerian philosophy adopted towards the management of the application is believed to have contributed to its popularity and success. A fertile environment was produced, developing student input and a positive interaction between group members, which may enhance future learning.

\section{Acknowledgements}

The author welcomes the opportunity to thank Simone Berg, who proved a most excellent moderator to the chat facility. Keith Smyth offered advice, without which this communication would not have been published. Anonymous reviewers have greatly enhanced the quality of this paper.

\section{References}

Anonymous (2004) Simple chat! 1.3. Available online at: http://www.hot-things.net (accessed 27 November 2005).

Baskerville, R. (1999) Investigating information systems with action research, Communications of the Association of Information Systems, 2, Article 19, 1-32.

Brass, K. \& Pilven, P. (1999) Using timely feedback on student progress to facilitate learning, presented at the Annual Conference of The Higher Education Research and Development Society of Australasia, Melbourne, Australia.

Buckner, K. \& Davenport, E. (2002) Teaching and learning in the VLCC: actions, reactions and emerging practice in a very large computing centre, in: S. Bagnara, S. Pozzi, A. Rizzo \& P Wright (Eds) 11th European Conference on Cognitive Ergonomics, Catania Sicily, 355-360.

Farkas, C., Ziegler, G., Meretei, A. \& Lörincz, A. (2002) Anonymity and accountability in selforganising electronic communities, in: Workshop on Privacy in the Electronic Society (WPES 2002), Washington, DC, 81-90.

Foulkes S. H. \& Anthony E. J. (1965) Group psychotherapy (2nd edn) (Harmondsworth, Pelican Books Ltd).

Halfpenny, P. \& Wellings, S. (2001) Can virtual seminars be used cost-effectively to enhance student learning?, Association for Learning Technology fournal, 9(3), 43-52.

Kay, D. (1998) Large introductory computer science classes: strategies for effective course management, in: Proceedings of the Twenty-Ninth SIGCSE Technical Symposium on Computer Science Education, Atlanta GA, 131-134.

Light, P., Colbourn, C. \& Light, V. (1997) Computer mediated tutorial support for conventional university courses, fournal of Computer Assisted Learning, 13, 228-235. 
Light V., Nesbit, E., Light, P. \& Burns, J. (2000) 'Let's you and me have a little discussion': computer mediated communication in support of campus-based university courses, Studies in Higher Education, 25(1), 85-96.

Mahoney, K. \& Baker, D. (2002) Elton Mayo and Carl Rogers: a tale of two techniques, fournal of Vocational Behaviour, 60, 437-450.

Millen, D. \& Patterson, J. (2003) Identity disclosure and the creation of social capital, in: Conference on Human Factors in Computing Systems (CHI 2003), Ft Lauderdale, FL, 720-721.

Pimintel, M., Fuks, H. \& Lucena, C. (2003) Co-text loss in chat tools, in: 4th International and Interdisciplinary Conference on Modelling and Using Context, Stanford, CA, 483-490.

Preece, J. (2000) Online communities: designing usability, supporting sociability (Chichester, John Wiley and Sons Ltd).

Preston, J. \& Shackelford, R. (1998) A system for improving distance and large scale classes, in: Proceedings of the 6th Annual Conference on the Teaching of Computing, Dublin, Ireland, 193-198.

Rogers, C. (1967) On becoming a person: a therapist's view of psychotherapy (London, Constable and Co. Ltd).

Salmon, G. (2002) E-tivities: the key to active online learning (London, Kogan Page).

Tolmie, A. and Boyle, J. (2000) Factors influencing the success of computer-mediated communication (CMC) environments in university teaching: a review and case study, Computers and Education, 34(2), 119-140.

Wilson, T. \& Whitelock, D. (1998) What are the perceived benefits of participating in a computermediated communication environment for distance learning computer science students?, Computers and Education, 30(3/4), 259-269.

Wright, N., Varey, A. \& Chesney, T. (2004) An investigation of sociability measurements in proximate online communities, in: IADIS International Conference on Web Based Communities, Lisbon, March.

\section{Appendix 1. The questionnaire}

1. Have you ever logged in to the WDM chat room $(\mathrm{y} / \mathrm{n})$ :

2. If not, please write something to help me understand why not.

3. Have you logged in and watched but never participated? If not, skip to (4). If so, please:

a) Say what you might have gained from watching as you did.

b) Say why you were not drawn to participate.

4. If you have participated:

a) What led you to try it?

b) What (if anything) have you learned about computing and WDM from it?

c) What (if anything) did you gain in a general social way?

d) Did the anonymity (nicknames) help or hinder? Should this be changed?

e) Overall, is the chat room worth keeping as part of the module, and why?

f) If it is kept, how could I make it better (and why)?

5. Is there anything else that might be useful to me that I forgot to ask? 